\title{
Surface Microenvironment Optimization Induced Robust Oxygen Reduction for Neutral Zinc-Air Batteries
}

\author{
Si Liu ${ }^{1}$, Han Cheng ${ }^{1}$, Jun Xia ${ }^{1}$, Chun Wang ${ }^{1}$, Renjie Gui ${ }^{1}$, Tianpei Zhou ${ }^{1}$, Hongfei Liu ${ }^{1}$, \\ Jing Peng ${ }^{1}$, Nan Zhang ${ }^{1}$, Wenjie Wang ${ }^{1}$, Wangsheng $\mathrm{Chu}^{1}$, Hengan $\mathrm{Wu}^{1}$, Changzheng $\mathrm{Wu}^{1}$, \\ and $\mathrm{Yi} \mathrm{Xie}^{1}$ \\ ${ }^{1}$ University of Science and Technology of China
}

February 10, 2021

\begin{abstract}
Neutral zinc-air batteries (ZABs) are promising candidates for the next-generation power devices with considerably elongated lifetime comparing to conventional alkaline ZABs. However, neutral cathodic oxygen reduction reaction is seriously limited by the mass transfer efficiency of hydroxyl due to insufficient interfacial chemical potential-gradient between catalytic layer and electrolyte. Herein, we highlight that electrochemical oxidation induced surface microenvironment optimization could realize optimal chemical potential-gradient around catalytic sites and bring outstanding neutral ORR activity. The electro-deposited sub-nano Pt decorated surface-microenvironment-optimized Co2N samples (denoted as Pt-SMO-Co2N NWs) possessed $92 \mathrm{mV}$ and $365 \mathrm{mV}$ lower overpotential than commercial $\mathrm{Pt} / \mathrm{C}$ and pristine Co2N in $0.2 \mathrm{M}$ PBS. As for neutral ZABs, Pt-SMOCo2N NWs cathode delivers a power density of $67.9 \mathrm{~mW}^{*} \mathrm{~cm}-2$ and displays negligible decay after nearly 80 hours stability test at $20 \mathrm{~mA}^{*} \mathrm{~cm}-2$. In depth characterization proposes that remarkable performance improvement originates from optimized microenvironment which increases the surface chemical potential gradient and facilitates proton coupled electron transfer during ORR. We anticipated that such synergetic optimization of microenvironment and intrinsic activity of active sites is an effective strategy which may be extended the catalytic reactions beyond ORR.
\end{abstract}

\section{Introduction}

With the depletion of non-renewable energy sources, the development of efficient energy storage technologies with low environmental impact becomes essential to realize energy and environment sustainability ${ }^{1-5}$. Zinc-air batteries (ZABs) is considered as one of the most promising candidates for the next-generation power devices due to their high theoretical energy density, low cost and environmental friendliness ${ }^{6-10}$. Comparing with conventional alkaline ZABs, neutral ZABs involve the same electrochemical reactions (aircathode: $\mathrm{O}_{2}+2 \mathrm{H}_{2} \mathrm{O}+4 \mathrm{e}^{-}=4 \mathrm{OH}^{-}$; zinc anode: $\mathrm{Zn}+2 \mathrm{OH}^{-}=\mathrm{ZnO}+\mathrm{H}_{2} \mathrm{O}+2 \mathrm{e}^{-}$) but are much more resistant to self-discharging induced zinc electrode corrosion and ambient $\mathrm{CO}_{2}$ absorption resulted electrolyte carbonation $^{11-13}$. However, the neutral media normally suffers from the insufficient ionic conductivity in electrolyte and the low chemical potential-gradient across the electrolyte/electrode interface ${ }^{14}$. Consequently, the oxygen reduction reaction (ORR), key electrochemical reactions at the air-cathode, are supposed to be kinetically more sluggish and possesses higher reaction barrier in neutral media than alkaline solution ${ }^{15}$. In this regard, it is crucial to develop highly active catalysts which can work efficiently and robustly in neutral environment to maximize the performance of ZABs.

During the last decade, plenty of efforts have been devoted to seeking various catalyst modification strategies for enhancing their ORR performance, including such as heteroatoms doping, molecular engineering, morphology regulation and so on ${ }^{16-18}$. Despite the regulation of intrinsic activity of catalytic materials, designing the local microenvironment around catalytic sites also plays a key role to realize enhanced catalytic 
performance ${ }^{19,20}$. Optimal microenvironment could realize the enrichment of intermediates across the electrolyte/electrode interface and further promote the interfacial chemical potential-gradient. With satisfied chemical potential-gradient, the intermediate energy states or even the reaction pathways can be tailored, which will decrease the reaction energy barrier and promote the reaction kinetics ${ }^{21,22}$. However, most of current work were focused on elevating the intrinsic activity of catalytic sites, yet there still lacks effective strategy to optimize the surface microenvironment around active sites ${ }^{23,24}$. Therefore, it remains a challenging but perspective route to improve the ZABs performance in neutral media through local microenvironment design of ORR catalysts.

Herein, we highlight that surface microenvironment optimization via electrochemical oxidation could serve as an effective way to design highly active ORR catalysts for air electrode of neutral ZABs. Owing to the synthetically tuning of both intrinsic catalytic activity and local microenvironment of the active sites, the prepared Pt-SMO- $\mathrm{Co}_{2} \mathrm{~N}$ NWs presented superior ORR activity in a $0.2 \mathrm{M}$ phosphate buffer solution at $\mathrm{pH}=$ 7.0 to pristine $\mathrm{Co}_{2} \mathrm{~N}$ NWs and commercial Pt/C. Moreover, the rechargeable ZABs based on Pt-SMO- $\mathrm{Co}_{2} \mathrm{~N}$ NWs and neutral electrolyte reached a power density of $67.9 \mathrm{~mW}^{*} \mathrm{~cm}^{2}$ and showed negligible decay during nearly 80 hours' stability test. Our work suggests that surface microenvironment optimization would be a new strategy to design advanced electrocatalysts for neutral ZABs, disclosing the pivotal mechanism of activating $\mathrm{H}_{2} \mathrm{O}$ and facilitating proton transfer process in ORR catalysis.

\section{Results and discussion}

To realize the synergetic optimization of intrinsic activity and microenvironment for active sites, electrochemical oxidation was conducted by applying a voltage from $0 \mathrm{~V}$ to $1.6 \mathrm{~V}$ on $\mathrm{Co}_{2} \mathrm{~N}$ nanowires in a $\mathrm{Pt}$ precursor contained solution (Figure 1a) . To follow the surface evolution of $\mathrm{Co}_{2} \mathrm{~N}$ nanowires, HRTEM was operated to study the surface microstructure of $\mathrm{Co}_{2} \mathrm{~N}$ before and after surface microenvironment optimization. Figure S3 shows the HRTEM image of pristine $\mathrm{Co}_{2} \mathrm{~N}$ nanowires with a lattice spacing value of $0.196 \mathrm{~nm}$ which could be ascribed to (120) plane. After the microenvironment optimization, a rough surface layer with small Pt clusters could be clearly observed in Figure 1b. High-angle annular dark-field scanning transmission electron microscopy (HAADF) further confirmed the formation of sub-size Pt clusters with a diameter of $1^{\sim} 2$ $\mathrm{nm}$ (the bright areas inFigure 1c ). The in-situ X-ray photoelectron spectra (XPS) equipped with Ar ions etching was operated to investigate the composition and electronic structure of the oxidized surface layer as a function of depth. As shown in Figure 1d, the fine-scanned Co $2 p$ XPS spectra of the pristine $\mathrm{Co}_{2} \mathrm{~N}$ nanowires and the Pt-SMO- $\mathrm{Co}_{2} \mathrm{~N}$ NWs can be well fitted with three different valence states (i.e. $\mathrm{Co}^{3+}$ at $795.2 \mathrm{eV}, \mathrm{Co}^{2+}$ at $797.0 \mathrm{eV}$, and $\mathrm{Co}^{0}$ at $\left.794.5 \mathrm{eV}\right)^{25,26}$ The filled area under each fitting curves represents the abundance of each valence state. The increased surface $\mathrm{Co}^{3+} / \mathrm{Co}^{0}$ ratio after surface microenvironment optimization suggests the formation of more $\mathrm{Co}^{3+}$ species through the oxidation of surface $\mathrm{Co}^{0} \mathrm{of}_{\mathrm{Co}_{2} \mathrm{~N}}$ nanowires, which is probably corresponding to high valence $\mathrm{CoOOH}$ layer according to previous studies ${ }^{26}$. With $3 \mathrm{keV}$ Ar etching, the ratio of $\mathrm{Co}^{3+} / \mathrm{Co}^{0}$ was decreased to nearly the same level with pristine $\mathrm{Co}_{2} \mathrm{~N}$ nanowires, indicating that the internal phase of $\mathrm{Co}_{2} \mathrm{~N}$ was maintained. Moreover, as shown in Figure 1e , two fitted peaks at $70.8 \mathrm{eV}$ and $74.1 \mathrm{eV}$ could be ascribed as the $\mathrm{Pt} 4 \mathrm{f}_{5 / 2}$ and $4 \mathrm{f}_{7 / 2}$ bands of $\mathrm{Pt}^{027}$. With $3 \mathrm{keV}$ Ar etching of surface layer, the peak intensity of $\mathrm{Pt} 4 \mathrm{f}_{5 / 2}$ and $4 \mathrm{f}_{7 / 2}$ was greatly reduced, suggesting that sub-nano Pt sites were deposited on the most surface layer after microenvironment optimization. 

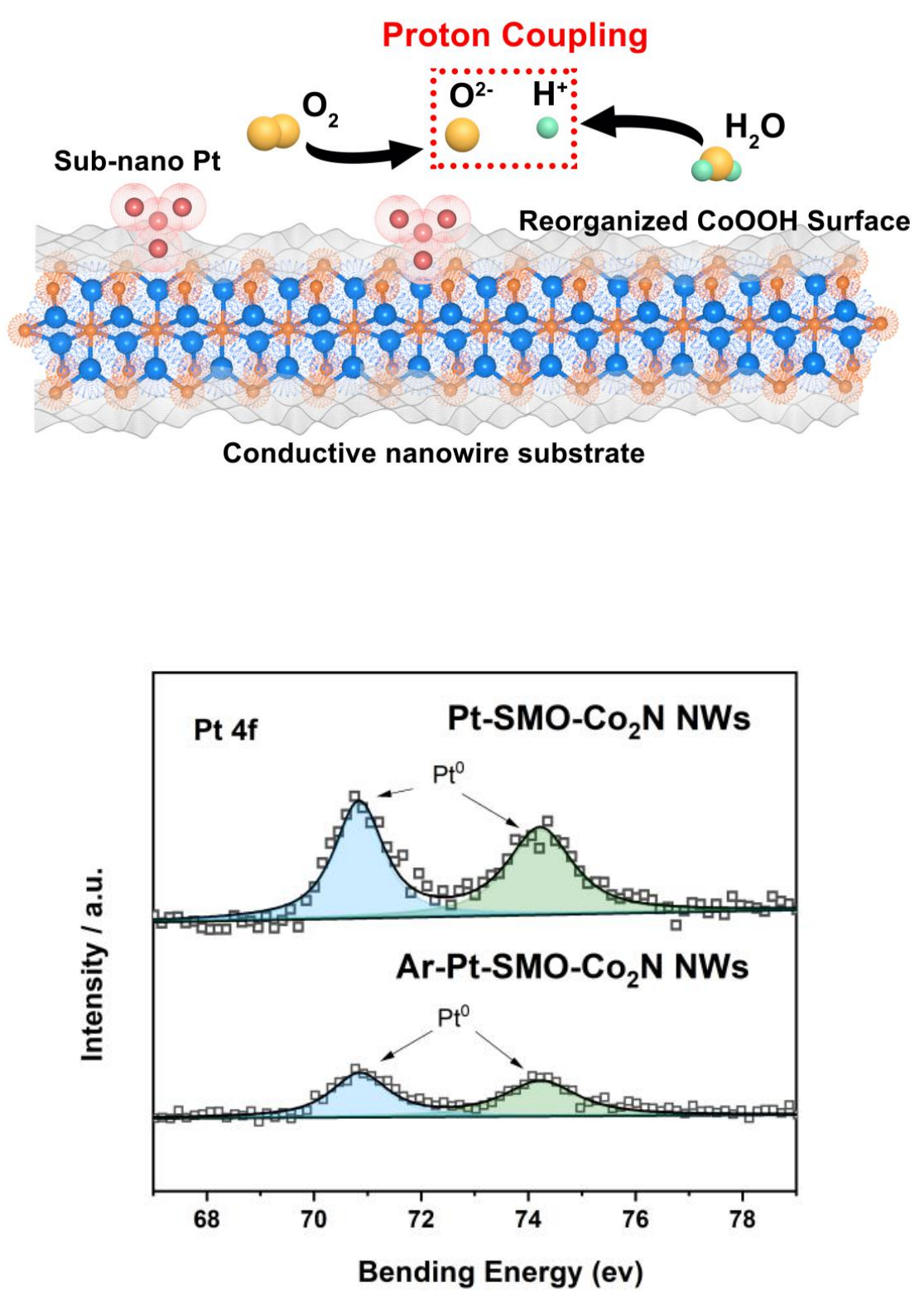

Figure 1 (a) Schematic illustration of synergistic surface optimization and proposed catalysis function. (b) HRTEM image of Pt-SMO-Co ${ }_{2} \mathrm{~N}$ NWs. (c) HADDF image of Pt-SMO-Co ${ }_{2} \mathrm{~N}$ NWs. (d) Co 2p XPS pattern of $\mathrm{Co}_{2} \mathrm{~N}$ NWs, Pt-SMO-Co ${ }_{2} \mathrm{~N}$ NWs and Ar-Pt-SMO-Co ${ }_{2} \mathrm{~N}$ NWs. (e) Pt 4 f XPS pattern of Pt-SMO-Co ${ }_{2} \mathrm{~N}$ NWs and Ar-Pt-SMO- $\mathrm{Co}_{2} \mathrm{~N}$ NWs.

To further investigate the local structure of cobalt and platinum in above catalysts, in-situ X-ray absorption fine structure (XAFS) was then employed to monitor the structure evolution during surface microenvironment optimization. Both Co K-edge and $\mathrm{Pt} \mathrm{L}_{3}$-edge spectra were recorded for the pristine electrocatalyst, and the electrocatalysts after surface microenvironment optimization for $40 \mathrm{~min}, 80 \mathrm{~min}$ and $120 \mathrm{~min}$. As shown in Figure 2a , despite a slight right shift toward higher absorption energy, the Co K-edge XANES spectra of the Pt-SMO- $\mathrm{Co}_{2} \mathrm{~N}$ NWs were still similar to pristine $\mathrm{Co}_{2} \mathrm{~N} \mathrm{NWs}$, suggesting that the bulk lattice framework 
was maintained. From the magnified spectra, a continuous right shift could be observed as the time increased from 0 to $120 \mathrm{~min}$, indicating the surface was gradually oxidized to higher valence during the electrochemical surface optimization. The Fourier transformed spectra of Co K-edge were shown in Figure 2b . During surface microenvironment optimization, EXAFS spectrums underwent a continuous decrease on Co- $\mathrm{N}$ bonds, while an increase of $\mathrm{Co}-\mathrm{O}$ bonds with the elongated electrochemical treatment, indicating the formation of oxyhydroxide layer ${ }^{26}$. In-situ XAFS was also operated to investigate local structure of Pt during surface microenvironment optimization at different stages (Figure 2c and 2d). The peak at $2.41 \AA$ could be ascribed to Pt-Pt bonds in Pt-SMO- $\mathrm{Co}_{2} \mathrm{~N}$ NWs, of which intensity increased with extended electrochemical oxidation duration. To illustrate the evolution of $\mathrm{Pt}$ local structure more straightforwardly, the wavelet transform (WT) analysis was performed (Figure 2e and 2f) ${ }^{28}$. The WT contour plots also showed the same trend as that of Fourier transformed Pt L-edge spectra. All these results clearly indicate the formation of sub-nano Pt cluster deposited on generated oxyhydroxide layer on the surface of $\mathrm{Co}_{2} \mathrm{~N} \mathrm{NWs}$.

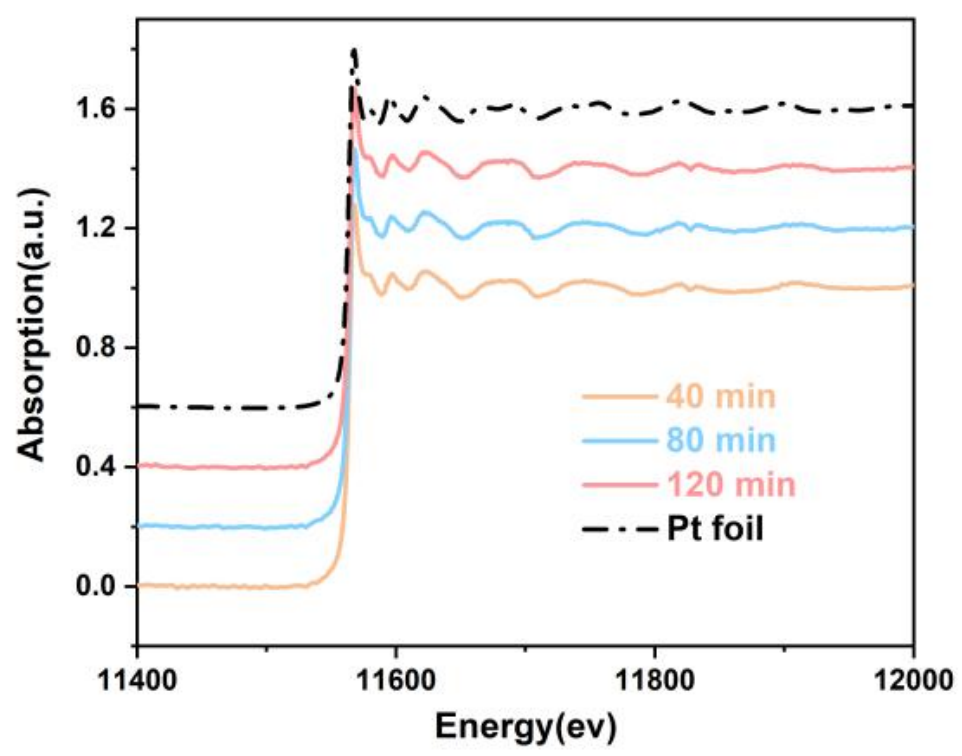




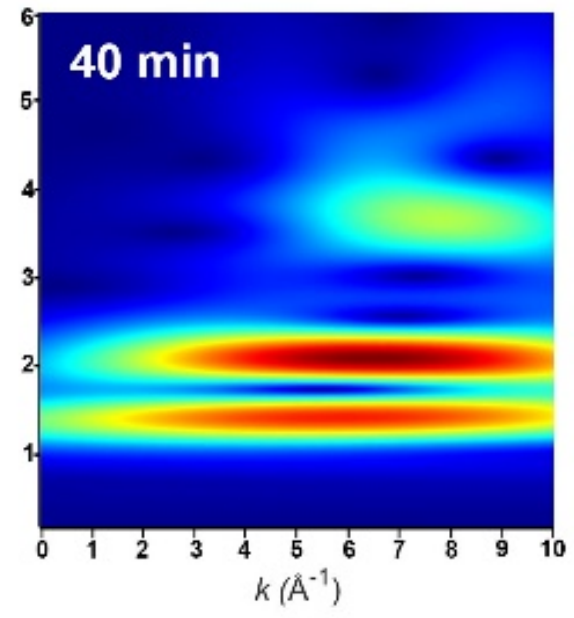




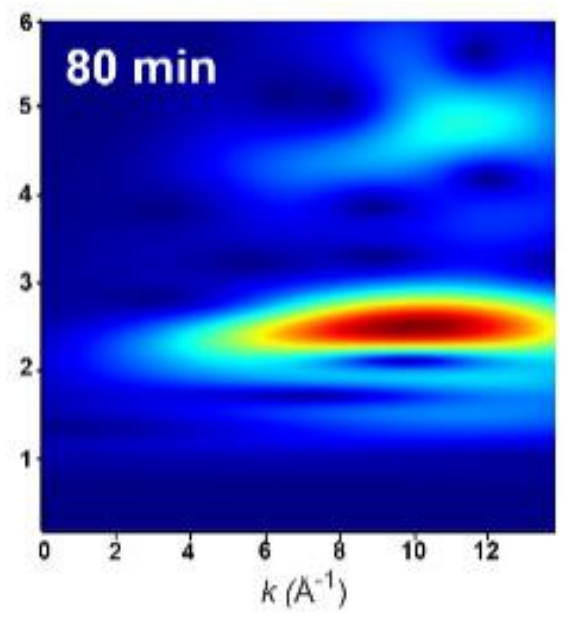

Figure 2 In-situ Co K near edge XANE spectra (a) and Pt L near edge XANE spectra (b) of Pt-SMO$\mathrm{CO}_{2} \mathrm{~N}$ NWs during synergistic surface microenvironment optimization process. FT-EXAFS spectra at the (c)Co K-edge and (d) Pt L-edge of Pt-SMO-Co ${ }_{2} \mathrm{~N}$ NWs. (e) Co K-edge WT-EXAFS contour plots and (f) Pt L-edge WT-EXAFS contour plots of Pt-SMO- $\mathrm{Co}_{2} \mathrm{~N}$ NWs during synergistic surface microenvironment optimization process.

To examine the catalytic effect of surface microenvironment optimization, the Pt-SMO- $\mathrm{Co}_{2} \mathrm{~N}$ NWs was used as working electrodes in $\mathrm{O}_{2}$-saturated $0.2 \mathrm{M}$ PBS $(\mathrm{pH}=7.0)$ to evaluate its electrocatalytic performance toward ORR. As illustrated inFigure 3a , the linear scan sweep voltammetry (LSV) curve exhibits the catalytic performance of Pt-SMO- $\mathrm{Co}_{2} \mathrm{~N}$ NWs with a positive onset potential of $0.960 \mathrm{~V}$ and a half-wave potential of $0.812 \mathrm{~V}$, much better than that of commercial Pt/C. Furthermore, the mass activity at $0.7,0.8$ and $0.9 \mathrm{~V}$ vs. RHE was calculated. As demonstrated in Figure $\mathbf{3 b}$, the mass activity of Pt-SMO- $\mathrm{Co}_{2} \mathrm{~N}$ NWs was almost doubled in comparison to commercial Pt/C. Moreover, the Pt mass activity of Pt-SMO$\mathrm{Co}_{2} \mathrm{~N}$ NWs is nearly 30 times higher than that of commercial $\mathrm{Pt} / \mathrm{C}$, excluding the contribution of $\mathrm{Co}_{2} \mathrm{~N}$. As shown in Figure 3c , Pt-SMO- $\mathrm{Co}_{2} \mathrm{~N}$ NWs possessed the lowest Tafel value, suggesting that the ORR kinetics on Pt-SMO- $\mathrm{Co}_{2} \mathrm{~N}$ NWs were obviously enhanced comparing to commercial Pt/C. To quantitatively understand the ORR activity of Pt-SMO-Co ${ }_{2} \mathrm{~N}$ NWs, detailed LSV tests were operated at different rotating speeds from 600 to $1600 \mathrm{rpm}$ (Figure 3d). The Koutecky-Levich (K-L) equation was used to calculate number of electrons transferred during the ORR process ${ }^{29}$. The electron-transfer number of Pt-SMO- $\mathrm{Co}_{2} \mathrm{~N}$ NWs is about 3.9 from $0.2-0.6 \mathrm{~V}$, suggesting the Pt-SMO- $\mathrm{Co}_{2} \mathrm{~N}$ NWs could catalyze oxygen reduction in a direct four-electron pathway under neutral conditions. Except for electrocatalytic activity, stability is also a key index to assess the performance of a given electrocatalyst. The polarization curves of Pt-SMO- $\mathrm{Co}_{2} \mathrm{~N}$ NWs recorded after 1000 cycles show no shift(Figure S4), indicating the excellent stability. Moreover, the 
electrocatalytic performance of OER was also assessed, as shown inFigure S5, Pt-SMO-Co 2 N NWs showed smallest overpotential at a current density of $10 \mathrm{~mA}^{*} \mathrm{~cm}^{-2}$, comparing to the $\mathrm{Co}_{2} \mathrm{~N}$ NWs and commercial $\mathrm{Pt} / \mathrm{C}$. In the light of the excellent bifunctional oxygen electrocatalytic property, Pt-SMO- $\mathrm{Co}_{2} \mathrm{~N} \mathrm{NWs}$ could serve as an ideal air-electrode of rechargeable zinc-air batteries (RZABs). Zn-Air battery was constructed by loading Pt-SMO- $\mathrm{Co}_{2} \mathrm{~N}$ NWs onto a gas diffusion layer (GDL) air electrode with a spray gun. A mixture aqueous solution of $4.0 \mathrm{M} \mathrm{NH}_{4} \mathrm{Cl}$ and $2.0 \mathrm{M} \mathrm{KCl}(\mathrm{pH}=7.0)$ was used as the electrolyte. For performance comparison, reference tests with equal loading of $\mathrm{Co}_{2} \mathrm{~N}$ and commercial $\mathrm{Pt} / \mathrm{C}$ catalysts on GDL were also conducted in the same manner. The polarization and power density curves are presented in Figure 3e , showing that Pt-SMO- $\mathrm{Co}_{2} \mathrm{~N}$ NWs possessed a comparable open-circle voltage with commercial $\mathrm{Pt} / \mathrm{C}$. The power density of Pt-SMO-Co ${ }_{2} \mathrm{~N}$ NWs eventually peaks at $67.9 \mathrm{~mW}^{*} \mathrm{~cm}^{-2}$, which is superior that of commercial Pt/C $\left(50 \mathrm{~mW}{ }^{*} \mathrm{~cm}^{-2}\right)$ and pristine $\mathrm{Co}_{2} \mathrm{~N} \mathrm{NWs}\left(22.5 \mathrm{~mW} * \mathrm{~cm}^{-2}\right)$. Chronoamperometric tests were then performed to evaluate the stability of the constructed RZABs. As shown in Figure $\mathbf{S 7}$, under the current density of $20 \mathrm{~mA}^{*} \mathrm{~cm}^{-2}$, it could maintain a stable voltage for nearly 80 hours, indicating the mitigated zinc corrosion in neutral environment. Moreover, the galvanostatic discharge-charge profile of Znair battery using Pt-SMO-Co $\mathrm{C}_{2} \mathrm{~N}$ NWs on GDL as air electrode was obtained under the current density of 2 $\mathrm{mA}^{*} \mathrm{~cm}^{-2}$. As expected, the cell voltage overpotential showed negligible change after $80 \mathrm{~h}$ cycles (Figure 3f) - Significantly, these results demonstrate that Pt-SMO- $\mathrm{Co}_{2} \mathrm{~N}$ NWs obtained by surface microenvironment optimization could serve as an excellent air electrode for RZABs which could deliver a high power density sustainably.

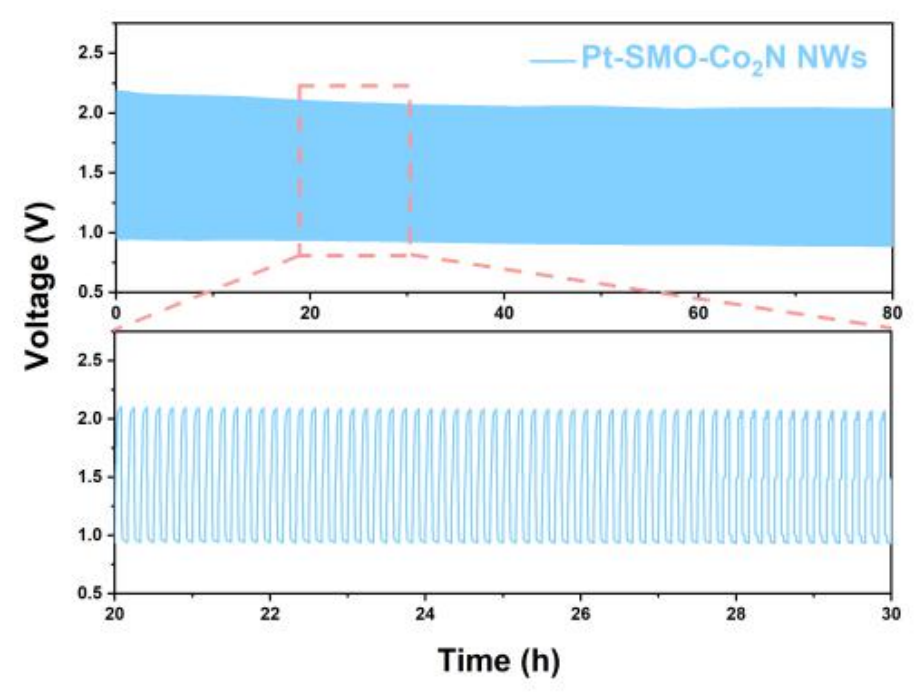

Figure 3 (a) ORR polarization curves of Pt-SMO- $\mathrm{Co}_{2} \mathrm{~N}$ NWs and reference catalysts in $0.2 \mathrm{M}$ PBS. (b) Corresponding mass activity comparison of Pt-SMO- $\mathrm{Co}_{2} \mathrm{~N}$ NWs and reference catalysts. (c) Tafel plots of Pt-SMO- $\mathrm{Co}_{2} \mathrm{~N}$ NWs and reference catalysts. (d) ORR polarization curves for Pt-SMO- $\mathrm{Co}_{2} \mathrm{~N} \mathrm{NWs}$ at different rotation speeds. (e) Polarization and power density curves of neutral ZABs with Pt-SMO- $\mathrm{Co}_{2} \mathrm{~N}$ NWs and reference catalysts as air-cathodes. (f) Galvanostatic discharge-charge cycling curves of the RZABs at $2 \mathrm{~mA}^{*} \mathrm{~cm}^{-2}$ with Pt-SMO- $\mathrm{Co}_{2} \mathrm{~N}$ NWs as air-cathodes.

To further understand the impact of surface microenvironment optimization for ORR, we constructed a Pt$\mathrm{CoOOH} @ \mathrm{Co}_{2} \mathrm{~N}$ model to simulate the composite structure of Pt-SMO- $\mathrm{Co}_{2} \mathrm{~N}$ NWs for thorectical study. DFT 
calculation was implemented to evaluate their surface energetics ${ }^{30-32}$. As shown inFigure $4 \mathbf{a}$, the Pt sites are highly active for binding $\mathrm{O}_{2}$ and the $\mathrm{Pt}-\mathrm{O}_{2}$ interaction is much stronger than $\mathrm{Pt}-\mathrm{H}_{2} \mathrm{O}$, indicating that the $\mathrm{Pt}$ sites prefer to bind $\mathrm{O}_{2}$ rather than $\mathrm{H}_{2} \mathrm{O}$. In contrast, $\mathrm{CoOOH}$ sites possess a notable higher affinity for $\mathrm{H}_{2} \mathrm{O}$ instead of $\mathrm{O}_{2}$ (Figure $4 \mathbf{b}$ ). Thus, it is supposed that $\mathrm{Pt}$ sites and $\mathrm{CoOOH}$ sites on the surface are responsible for binding different adsorbates, preferentially yielding $\mathrm{Pt}-\mathrm{O}_{2}$ and $\mathrm{Co}-\mathrm{OH}_{2}$ species respectively. On the basis of above adsorption model, DFT calculations further suggested the capability of breaking the $\mathrm{O}-\mathrm{O}$ bond and $\mathrm{O}-\mathrm{H}$ bond at catalytic sites and the dissociation of $\mathrm{H}_{2} \mathrm{O}$ is energetically highly favorable on the $\mathrm{CoOOH}$ sites while the $\mathrm{O}_{2}$ dissociation is favored on the Pt sites. Consequently, it is suggested that the constructed model of surface $\mathrm{CoOOH}$ layer with deposited $\mathrm{Pt}$ cluster possess synergistic surface for ORR catalysis. With the Pt sites binding and cleaving $\mathrm{O}_{2}$ and the $\mathrm{CoOOH}$ sites enriching and activating $\mathrm{H}_{2} \mathrm{O}$, the proton-coupled electron transfer process of oxygen reduction could be significantly facilitated. Based on the above results, the proposed synergistic mechanism of the Pt-SMO- $\mathrm{Co}_{2} \mathrm{~N}$ NWs catalyzed ORR is illustrated stepwise in Figure 4c . Considering Pt- $\mathrm{OH}$ and $\mathrm{Co}-\mathrm{OH}$ as the initial states, the $\mathrm{O}_{2}$ would preferentially binds to the $\mathrm{Pt}$ site after the detachment of an $\mathrm{OH}^{-}$group, yielding a $\mathrm{Pt}-\mathrm{O}_{2}$ superoxide intermediate. Meanwhile, a $\mathrm{H}_{2} \mathrm{O}$ molecule attaches to the Co site to generate yield a $\mathrm{Co}-\mathrm{OH}_{2}$ species. Afterwards, surface proton transfer would occur between $\mathrm{Co}-\mathrm{OH}_{2}$ and $\mathrm{Pt}-\mathrm{O}_{2}$, leading to the formation of $\mathrm{Co}-\mathrm{OH}$ and $\mathrm{Pt}-\mathrm{OOH}$ peroxide intermediates. Followed by two further electron reduction, Pt-OOH species would turn into Pt-O by releasing an $\mathrm{OH}^{-}$group. The second proton transfer process from $\mathrm{Co}-\mathrm{OH}_{2}$ to the generated $\mathrm{Pt}-\mathrm{O}$ would then proceed to regenerate the $\mathrm{Pt}-\mathrm{OH}$. Overall, the proposed mechanism is mainly constituted by two aspects: the proton mediation rising from turnover of $\mathrm{Co}-\mathrm{OH} / \mathrm{Co}-\mathrm{OH}_{2}$ and the proton transfer between contiguous $\mathrm{Co}$ and $\mathrm{Pt}$ sites. The above theoretical observations clearly demonstrated that synergistic active sites and optimized surface microenvironment could mediate the transportation of intermediate species to accelerate the reaction kinetics.

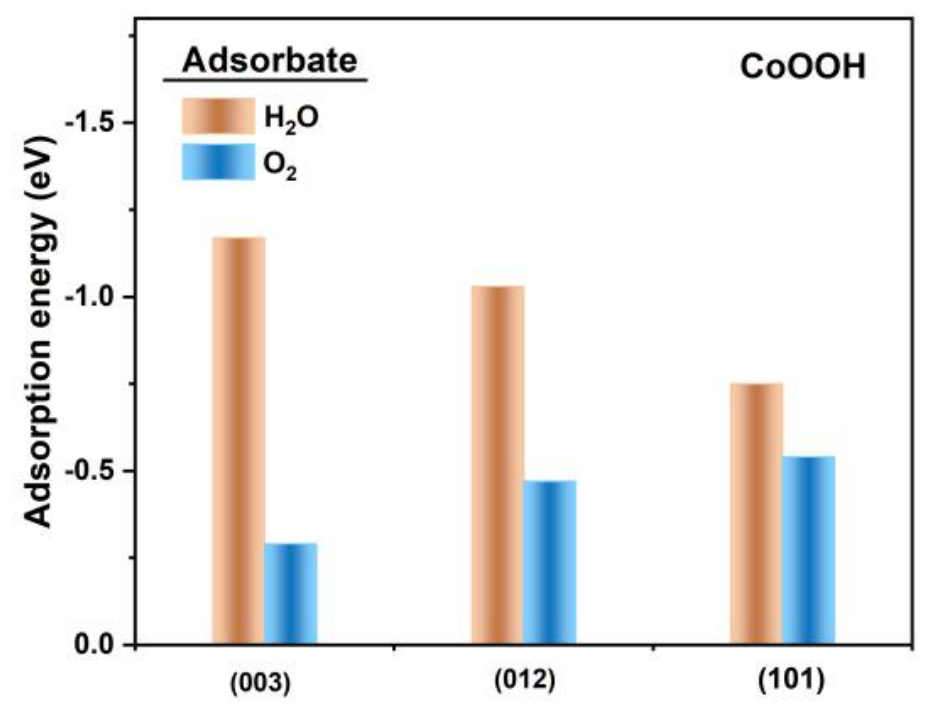




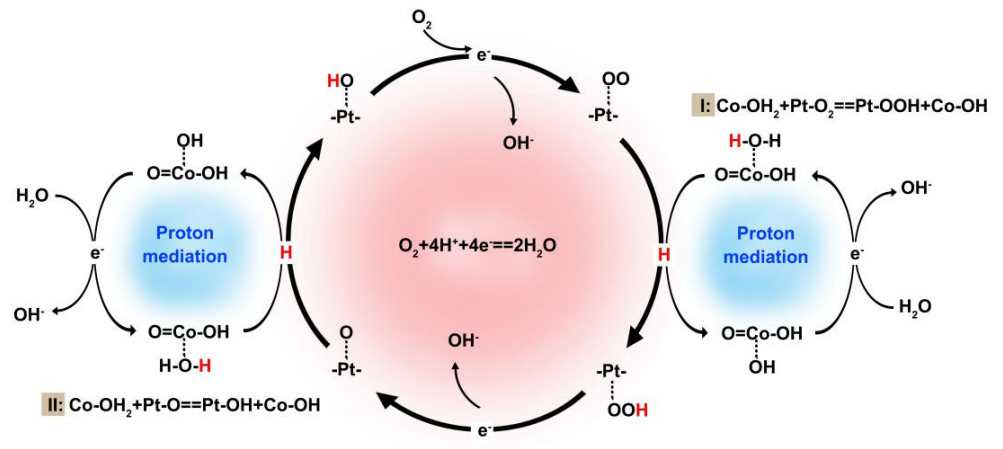

Figure 4 (a) Density functional theory (DFT) calculated adsorption energies for $\mathrm{H}_{2} \mathrm{O}$ and $\mathrm{O}_{2}$ on the $\mathrm{Pt}$ sites of (100), (110) and (111) surface. (b) Density functional theory (DFT) calculated adsorption energies for $\mathrm{H}_{2} \mathrm{O}$ and $\mathrm{O}_{2}$ on the CoOOH sites of (003), (012) and (101) surface. (c) Schematic illustration of the proposed synergistic mechanism of ORR on Pt-SMO- $\mathrm{Co}_{2} \mathrm{~N}$ NWs, including the dissociative reduction of $\mathrm{O}_{2}$ at the Pt site, the proton mediation by the $\mathrm{CoOOH}$ site, and the surface proton transfer in between (reactions I and II).

\section{Conclusions}

In summary, we have demonstrated surface microenvironment optimization as an effective way to design highly active ORR catalyst, serving as air electrode for neutral ZABs. Benefiting from the optimal surface microenvironment induced synergistic effects between different active sites, the achieved Pt-SMO- $\mathrm{Co}_{2} \mathrm{~N} \mathrm{NWs}$ presented extraordinary ORR activity. In $2.0 \mathrm{M} \mathrm{PBS} \mathrm{(pH=7.0),} \mathrm{Pt-SMO-Co} 2 \mathrm{~N}$ NWs showed a positive onset potential of $0.960 \mathrm{~V}$ and a half-wave potential of $0.812 \mathrm{~V}$, which is $92 \mathrm{mV}$ higher than that of commercial $\mathrm{Pt} / \mathrm{C}$. The power density of neutral ZABs taking Pt-SMO- $\mathrm{Co}_{2} \mathrm{~N}$ NWs as cathode catalyst could reach 67.9 $\mathrm{mW}^{*} \mathrm{~cm}^{-2}$, outperforming commercial $\mathrm{Pt} / \mathrm{C}$ under the same circumstance and displayed barely decay after 80 hours' discharge-charge test. Moreover, based on the ideal material platform built on Pt-SMO- $\mathrm{Co}_{2} \mathrm{~N}$ NWs, in-depth characterization and mechanistic understanding of ORR was disclosed. Our work reveals a new strategy for the ORR catalyst design through the construction of optimal surface microenvironment and offers new insights toward the key role of activating $\mathrm{H}_{2} \mathrm{O}$ and facilitating proton transfer process in ORR catalysis.

\section{ASSOCIATED CONTENT}

XRD patterns, TEM images and additional electrochemical date are included in the supporting information. This material is available free of charge via the Internet at http://pubs.acs.org.

\section{AUTHOR INFORMATION}

Corresponding Author

czwu@ustc.edu.cn;

yxie@ustxc.edu.cn

Author Contributions

$\S$ These authors contributed equally. 
Notes The authors declare no competing financial interests.

\section{ACKNOWLEDGMENT}

This work was financially supported by the National Basic Research Program of China (2017YFA0206702), Natural Science Foundation of China (No. 21925110, 21890751), China Postdoctoral Science Foundation (2019TQ0299) and Fundamental Research Funds for the Central Universities (No. WK 2060190084, No. WK 5290000001). The authors thank Dr Jie Tian and Dr Huijuan Wang at Engineering and Materials Science Experiment Centre for the help of HRTEM experiments. The authors also appreciate the support from the Major/Innovative Program of Development Foundation of Hefei Center for Physical Science and Technology. This work was partially carried out at the USTC Center for Micro and Nanoscale Research and Fabrication.

\section{REFERENCES}

1. Larcher, D. \& Tarascon, J. M. Towards greener and more sustainable batteries for electrical energy storage. Nat. Chem. 7 , 19-29, doi:10.1038/nchem.2085 (2015).

2. Aricò, A. S., Bruce, P., Scrosati, B., Tarascon, J.-M. \& van Schalkwijk, W. Nanostructured materials for advanced energy conversion and storage devices. Nat. Mater. 4 , 366-377, doi:10.1038/nmat1368 (2005).

3. Dresselhaus, M. S. \& Thomas, I. L. Alternative energy technologies.Nature 414 , 332-337, doi:10.1038/35104599 (2001).

4. Suntivich, J. et al. Design principles for oxygen-reduction activity on perovskite oxide catalysts for fuel cells and metal-air batteries.Nat. Chem. 3 , 546-550, doi:10.1038/nchem.1069 (2011).

5. Debe, M. K. Electrocatalyst approaches and challenges for automotive fuel cells. Nature 486 , 43-51, doi:10.1038/nature11115 (2012).

6. Tang, C., Wang, B., Wang, H.-F. \& Zhang, Q. Defect engineering toward atomic Co-Nx-C in hierarchical graphene for rechargeable flexible solid Zn-air batteries. Adv. Mater. 29 , 1703185, doi:10.1002/adma.201703185 (2017).

7. Yu, P. et al. Co Nanoislands rooted on $\mathrm{Co}-\mathrm{N}-\mathrm{C}$ nanosheets as efficient oxygen electrocatalyst for Zn-air batteries. Adv. Mater. 31 , 1901666, doi:10.1002/adma.201901666 (2019).

8. Meng, F., Zhong, H., Bao, D., Yan, J. \& Zhang, X. In situ coupling of strung $\mathrm{Co}_{4} \mathrm{~N}$ and intertwined N-C fibers toward free-standing bifunctional cathode for robust, efficient, and flexible $\mathrm{Zn}$-air batteries. J. Am. Chem. Soc. 138 , 10226-10231, doi:10.1021/jacs.6b05046 (2016).

9. Jiang, Y. et al. Interpenetrating triphase cobalt-based nanocomposites as efficient bifunctional oxygen electrocatalysts for long-lasting rechargeable Zn-air batteries. Adv. Energy. Mater.8 , 1702900, doi:10.1002/aenm.201702900 (2018).

10. Tong, Y. et al. A bifunctional hybrid electrocatalyst for oxygen reduction and evolution: cobalt oxide nanoparticles strongly coupled to B, N-decorated graphene. Angew. Chem. Int. Ed.56 , 7121-7125, doi:10.1002/anie.201702430 (2017).

11. Li, Y. \& Dai, H. Recent advances in zinc-air batteries.Chem. Soc. Rev. 43 , 5257-5275, doi:10.1039/C4CS00015C (2014).

12. Xia, B. Y. et al. A metal-organic framework-derived bifunctional oxygen electrocatalyst. Nat. Energy. 1 , 15006, doi:10.1038/nenergy.2015.6 (2016).

13. Sumboja, A. et al. Durable rechargeable zinc-air batteries with neutral electrolyte and manganese oxide catalyst. J. Power Sources. 332, 330-336, doi:10.1016/j.jpowsour.2016.09.142 (2016).

14. Su, Y. et al. A highly efficient catalyst toward oxygen reduction reaction in neutral media for microbial fuel cells. Ind. Eng. Chem. Res. 52, 6076-6082, doi:10.1021/ie4003766 (2013). 
15. Clark, S., Latz, A. \& Horstmann, B. Rational development of neutral aqueous electrolytes for zinc-air batteries. ChemSusChem.10 , 4735-4747, doi:10.1002/cssc.201701468 (2017).

16. Xie, L. et al. Molecular engineering of a 3D self-supported electrode for oxygen electrocatalysis in neutral media. Angew. Chem. Int. Ed. 58 , 18883-18887, doi:10.1002/anie.201911441 (2019).

17. Jung, J.-I., Jeong, H. Y., Lee, J.-S., Kim, M. G. \& Cho, J. A bifunctional perovskite catalyst for oxygen reduction and evolution.Angew. Chem. Int. Ed. 53 , 4582-4586, doi:10.1002/anie.201311223 (2014).

18. Ma, T. Y., Ran, J., Dai, S., Jaroniec, M. \& Qiao, S. Z. Phosphorus-Doped Graphitic Carbon Nitrides Grown In Situ on Carbon-Fiber Paper: Flexible and Reversible Oxygen Electrodes. Angew. Chem. Int. Ed. 54, 4646-4650, doi:10.1002/anie.201411125 (2015).

19. Gong, M. et al. Nanoscale nickel oxide/nickel heterostructures for active hydrogen evolution electrocatalysis.Nat. Commun. 5 , 4695, doi:10.1038/ncomms5695 (2014).

20. Lu, X. F., Chen, Y., Wang, S., Gao, S. \& Lou, X. W. Interfacing manganese oxide and cobalt in porous graphitic carbon polyhedrons boosts oxygen electrocatalysis for $\mathrm{Zn}$-air batteries. Adv. Mater.31 , 1902339, doi:10.1002/adma.201902339 (2019).

21.Xing, Z., Hu, L., Ripatti, D. S., Hu, X. \& Feng, X. Enhancing carbon dioxide gas-diffusion electrolysis by creating a hydrophobic catalyst microenvironment. Nat. Commun. 12, 136, doi:10.1038/s41467-02020397-5 (2021).

22. Guo, C. et al. Engineering High-Energy Interfacial Structures for High-Performance Oxygen-Involving Electrocatalysis. Angew. Chem. Int. Ed. 56 , 8539-8543, doi:10.1002/anie.201701531 (2017).

23. Yu, L., Yi, Q., Li, G., Chen, Y. \& Yang, X. FeCo-Doped Hollow Bamboo-Like C-N Composites as Cathodic Catalysts for Zinc-Air Battery in Neutral Media. J. Electrochem. Soc 165 , A2502-A2509, doi:10.1149/2.0481811jes (2018).

24. Shao, M., Chang, Q., Dodelet, J.-P. \& Chenitz, R. Recent Advances in Electrocatalysts for Oxygen Reduction Reaction. Chem. Rev.116 , 3594-3657, doi:10.1021/acs.chemrev.5b00462 (2016).

25. Jin, H. et al. In situ Cobalt-Cobalt Oxide/N-Doped Carbon Hybrids As Superior Bifunctional Electrocatalysts for Hydrogen and Oxygen Evolution. J. Am. Chem. Soc. 137, 2688-2694, doi:10.1021/ja5127165 (2015).

26. Liu, S. et al. Dual Modulation via Electrochemical Reduction Activiation on Electrocatalysts for Enhanced Oxygen Evolution Reaction.ACS Energy Lett. 4 , 423-429, doi:10.1021/acsenergylett.8b01974 (2019).

27. Kerrec, O., Devilliers, D., Groult, H. \& Marcus, P. Study of dry and electrogenerated Ta2O5 and Ta/Ta2O5/Pt structures by XPS.Mater. Sci. Eng. B. 55 , 134-142, doi:https://doi.org/10.1016/S09215107(98)00177-9 (1998).

28. Chen, Y. et al. Atomic-Level Modulation of Electronic Density at Cobalt Single-Atom Sites Derived from Metal-Organic Frameworks: Enhanced Oxygen Reduction Performance. Angew. Chem. Int. Ed. doi: 10.1002/anie.202012798.

29. Zhou, T. et al. Ultrathin Cobalt Oxide Layers as Electrocatalysts for High-Performance Flexible Zn-Air Batteries.Adv. Mater. 31, 1807468, doi:10.1002/adma.201807468 (2019).

30. Wang, Y. et al. Synergistic Mn-Co catalyst outperforms Pt on high-rate oxygen reduction for alkaline polymer electrolyte fuel cells.Nat. Commun. 10 , 1506, doi:10.1038/s41467-019-09503-4 (2019).

31. Subbaraman, R. et al. Trends in activity for the water electrolyser reactions on $3 \mathrm{~d} \mathrm{M}(\mathrm{Ni}, \mathrm{Co}, \mathrm{Fe}, \mathrm{Mn})$ hydr(oxy)oxide catalysts.Nat. Mater. 11 , 550-557, doi:10.1038/nmat3313 (2012). 
32. Xu, K. et al. Controllable Surface Reorganization Engineering on Cobalt Phosphide Nanowire Arrays for Efficient Alkaline Hydrogen Evolution Reaction. Adv. Mater. 30 , 1703322, doi:10.1002/adma.201703322 (2018).

\section{Hosted file}

manuscript.pdf available at https://authorea.com/users/394967/articles/508313-surfacemicroenvironment-optimization-induced-robust-oxygen-reduction-for-neutral-zinc-airbatteries 